\title{
Local Density Variations in Planetary Nebulae
}

\author{
M. J. Seaton \\ University College, University of London, London, England
}

$\mathrm{D}^{\mathrm{n}}$ ENSITIES in ionized nebulae may be estimated from the surface brightness, which essentially gives an rms density,

$$
n_{e}^{(\mathrm{rms})}=\left[\frac{1}{V} \int n_{e}^{2} d V\right]^{\frac{1}{2}}
$$

Independent estimates may be obtained from deactivation rates deduced from relative forbidden line intensities. At the second Symposium ${ }^{1}$ it was suggested that differences in the results obtained might be due to local density variations. Since that time, greatly improved brightness measurements have been made $^{2}$ and

TABLE I.

\begin{tabular}{lccc}
\hline \hline \multicolumn{1}{c}{$n_{\mathrm{o}}^{(1)}$} & $n_{\mathrm{e}}(2)$ & $V_{2} / V_{1}$ & $n_{\mathrm{e}}$ (rms) \\
\hline$\rightarrow 0$ & $3.5 \times 10^{4}$ & $\rightarrow 0$ & $\rightarrow 0$ \\
$2.5 \times 10^{3}$ & $4.6 \times 10^{4}$ & 0.024 & $0.75 \times 10^{4}$ \\
$5.0 \times 10^{3}$ & $7.3 \times 10^{4}$ & 0.0155 & $1.03 \times 10^{4}$ \\
$7.5 \times 10^{3}$ & $23.5 \times 10^{4}$ & 0.0016 & $1.20 \times 10^{4}$ \\
$8.5 \times 10^{3}$ & $\rightarrow \infty$ & $\rightarrow 0$ & $1.26 \times 10^{4}$ \\
& & & \\
\hline
\end{tabular}

TABLE II.

\begin{tabular}{llll}
\hline \hline NGC & a & b $^{10^{-4} \times n_{e}}$ & \multicolumn{1}{c}{$c$} \\
\hline 6543 & 2.4 & 1.5 & 0.6 \\
6572 & 4 & 1.9 & 1.0 \\
7009 & 2.8 & 1.0 & 0.6 \\
7027 & 4.5 & 1.1 & 0.85 \\
7662 & 5 & 1.2 & 0.44 \\
\hline \hline
\end{tabular}

- Density from deactivation methods other than $3729 / 3726$.

b Density from surface brightness.

1 M. J. Seaton, Gas Dynamics of Cosmic Clouds, edited by H. C. van de Hulst and J. M. Burgers (North Holland Publishing Company, Amsterdam, 1955), p. 75.

${ }^{2}$ W. Liller and L. H. Aller, Astrophys. J. 120, 48 (1954); W. Liller, Astrophys. J. 122, 240 (1955). further accurate line intensities obtained., ${ }^{3,4}$ An additional deactivation method has been developed ${ }^{5}$ in which use is made of the measured Balmer discontinuity.

Densities deduced from various deactivation effects are all in fair accord, ${ }^{5,6}$ with the exception of those obtained $^{7}$ from the [OII] 3729/3726 ratio, which are considerably smaller than the others. The explanation is that the 3729,3726 lines have very small transition probabilities and will therefore be stronger relative to less highly forbidden lines in low density regions. For NGC 7027, models have been assumed ${ }^{7}$ for which $n_{e}$ is equal to $n_{e}^{(1)}$ in a volume $V_{1}$ and to $n_{e}^{(2)}$ in a volume $V_{2}$, where $V_{1}+V_{2}=V$. The following values of $n_{e}^{(1)}, n_{e}^{(2)}$ and $V_{2} / V_{1}$ are consistent with the observed [OII] ratios $3729 / 3726$ and $(3729+3726) /(7320+7330)$ (see Table I). The deduced values of $n_{e}{ }^{(\mathrm{rms})}$ are in satisfactory agreement with the value $n_{e}^{(\mathrm{rms})}=1.1 \times 10^{4} \mathrm{~cm}^{-3} \mathrm{ob}-$ tained from the surface brightness using a revised extinction correction. ${ }^{8}$ It may be considered that $n_{e}=6 \times 10^{3} \mathrm{~cm}^{-3}$ in $99 \%$ of the volume and $n_{e}=9 \times 10^{4}$ $\mathrm{cm}^{-3}$ in $1 \%$ of the volume.

For other nebulae the results shown in Table II have been obtained. ${ }^{7,9}$ In all cases the differences in the densities obtained by the three methods are such as to suggest the existence of local condensations. Condensation effects seem to be greatest for NGC 7662, which is very irregular in appearance. The only planetary considered $^{7}$ for which there is no evidence for purely local density fluctuations, is the particularly uniform object IC 418.

\footnotetext{
${ }^{3}$ Aller, Bowen, and Minkowski, Astrophys. J. 122, 62 (1955).

${ }^{4}$ R. Minkowski and L. H. Aller, Astrophys. J. 124, 93 (1956).

5 M. J. Seaton, Monthly Notices Roy. Astron. Soc. 115, 279 (1955).

6 M. J. Seaton, Monthly Notices Roy. Astron. Soc. 114, 154 (1954).

${ }^{7}$ M. J. Seaton and D. E. Osterbrock, Astrophys. J. 125, 66 (1957).

${ }^{8}$ L. H. Aller and R. Minkowski, Astrophys. J. 124, 110 (1956).

${ }^{9}$ L. H. Aller, Astrophys. J. 125, 84 (1957).
} 